\title{
The endomorphism ring of an indecomposable module with an Artinian projective cover
}

\author{
By Tsutomu Takeuchi
}

As is well known, each endomorphism of an Artinian, Noetherian and indecomposable module is either nilpotent or an automorphism. C. I. Vinsonhaler has proved that each endomorphism of an indecomposable module with a Noetherian injective hull is either nilpotent or an automorphism (see [6], [7] and [5; p. 75]). In this note we shall verify that each endomorphism of an indecomposable module with an Artinian projective cover is either nilpotent or an automorphism.

We will assume throughout that $M$ denotes a nonzero unital left $R$ module, where $R$ is a nonzero ring with identity.

Theorem. Let $M$ be an indecomposable left $R$-module and let $P$ be an Artinian left $R$-module which is a projective cover of $M$ with a minimal epimorphism $\varphi: P \rightarrow M$. Then each endomorphism $\alpha$ of $M$ is either nilpotent or an automorphism.

Remark $1 . M$ is called a semiperfect module iff every factor module of $M$ has a projective cover (cf. Mares [4]).

Let $N$ be a submodule of $M$. A cocomplement $N^{c}$ of $N$ in $M$ is a minimal submodule of $M$ such that $N+N^{c}=M . \quad M$ is called a cocomplemented module iff every submodule of $M$ has a cocomplement in $M$.

As is easily proven, semiperfect modules are cocomplemented. Conversely, projective cocomplemented modules are semiperfect (see KaschMares [3]).

REMARK 2. In the following commutative diagram with left $R$-modules $M_{i}$ and homomorphisms $\phi_{i}(i=1,2,3)$ :

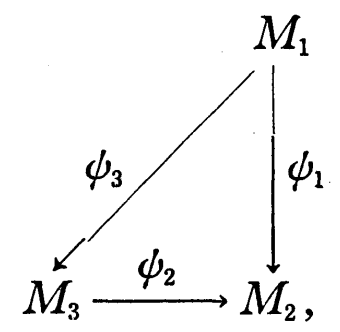

if $\phi_{1}$ is an epimorphism and if $\psi_{2}$ is a minimal epimorphism, then $\psi_{3}$ is necessarily an epimorphism. 
Proof of Theorem. Since also $M$ is Artinian, there exists a positive integer $r$ such that $M \alpha^{r}=M \alpha^{r+1}=\cdots$. Evidently, every Artinian module is cocomplemented, so that $P$ is semiperfect (Remark 1). Then, so is $M$ since every factor module of a semiperfect module is semiperfect. Put $M_{0}$ $=\operatorname{Ker} \alpha^{r}$ and let $P_{0}$ be a projective cover of $M / M_{0}$ with a minimal epimorphism $\varphi_{0}: P_{0} \longrightarrow M / M_{0}$. If $\pi: M \longrightarrow M / M_{0}$ is the natural epimorphism, then there exists a homomorphism $\psi_{0}: P \longrightarrow P_{0}$ such that $\psi_{0} \varphi_{0}=\varphi \pi$, by the projectivity of $P$. But $\phi_{0}$ is an epimorphism (Remark 2), whence $P_{0}$ is Artinian. Since $M_{0} \alpha \subset M_{0}, \alpha$ induces the endomorphism $\bar{\alpha}$ of $M / M_{0}$ by $\left(a+M_{0}\right) \bar{\alpha}=a \alpha+M_{0}(a \in M)$. Moreover, $M \alpha^{r}=M \alpha^{r+1}$ asserts that $\bar{\alpha}$ is an epimorphism. Then we have, by the projectivity of $P_{0}$, an endomorphism $\phi$ of $P_{0}$ such that $\phi \varphi_{0}=\varphi_{0} \bar{\alpha}$.

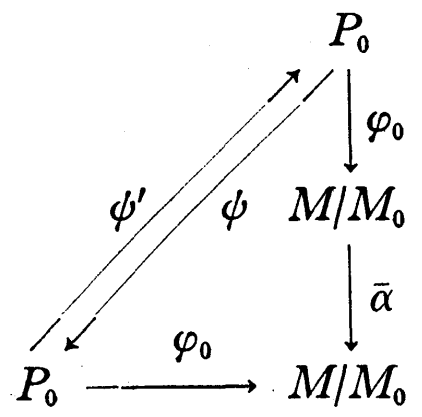

But $\psi$ is an epimorphism (again by Remark 2), so that the projective $P_{0}$ has a splitting endomorphism $\psi^{\prime} ; \psi^{\prime} \phi$ is the identity mapping of $P_{0}$. Since $P_{0}$ is Artinian, there exists a positive integer $s$ such that $P_{0} \phi^{\prime s}=P_{0} \psi^{\prime s+1}$. Since $\psi^{\prime}$ is monomorphic, we obtain $P_{0}=P_{0} \psi^{\prime}$, so that $\left(\psi^{\prime}\right.$ or $) \psi$ is an isomorphism.

Now, put $K=\operatorname{Ker} \varphi_{0} \bar{\alpha}$. Considering that $K \psi$ is included in $K$ which is also Artinian, we know the existence of a positive integer $t$ satisfying $K \psi^{t}=K \psi^{t+1}$. The monomorphism $\psi$ deduces $K=K \psi$. Then, this yields that $\bar{\alpha}$ is a monomorphism. Next, let $a$ be an arbitrary element of Ker $\alpha^{2 r}$. Then, $a \alpha^{r}$ is in $M_{0}$ and hence $\left(a+M_{0}\right) \bar{\alpha}^{r}=0$. Since $\bar{\alpha}$ is monomorphic, $a$ is contained in $M_{0}$, showing that $\operatorname{Ker} \alpha^{r}=\operatorname{Ker} \alpha^{2 r}$. This implies, together with $M \alpha^{r}=M \alpha^{2 r}$, that $M$ is the direct sum of $M \alpha^{r}$ and $\operatorname{Ker} \alpha^{r}$. It follows from the assumption of $M$ to be indecomposable that $M \alpha^{r}=0$ or that $M \alpha^{r}=M$ and $\operatorname{Ker} \alpha^{r}=0$. Consequently, $\alpha$ is nilpotent or $\alpha^{r}$ is, and hence, $\alpha$ is an automorphism, as required. 


\section{References}

[1] J. W. FISHER: Nil subrings of endomorphism rings of modules, Proc. Amer. Math. Soc. 34 (1972), 75-78.

[2] J. W. Fisher: Finiteness conditions for projective and injective modules, Proc. Amer. Math. Soc. 40 (1973), 389-394.

[3] FR. KASCH and E. A. MARES: Eine Kennzeichnung semi-perfekter Moduln, Nagoya Math. J. 27 (1966), 525-529.

[4] E. A. MARES: Semi-perfect modules, Math. Zeitschr. 82 (1963), 347-360.

[5] H. TACHIKaWa: Quasi-Frobenius Rings and Generalizations, Lecture Notes in Mathematics, No. 351, Springer (1973).

[6] C. I. VinsonhaleR: Orders in QF-3 rings, J. Algebra 14 (1970), 83-90.

[7] C. I. VinsonhaleR: Supplement to the paper, "Orders in QF-3 rings", $J$. Algebra 17 (1971), 149-151.

(Received June 18, 1974) 